\title{
Retos y oportunidades en la implementación del tamiz neonatal para fibrosis quística
}

\section{Challenges and opportunities in the implementation of the neonatal screening for cystic fibrosis}

Isabel Ibarra-González, ${ }^{1}$ Gabriel Gutiérrez-Morales, ${ }^{2}$ Marcela Vela-Amieva, ${ }^{3}$ José Ariel Castillo-Mogel, ${ }^{4}$ Luz del Alba Herrera-Pérez, ${ }^{4}$ Guillermo Caamal-Parra, ${ }^{4}$ Nazarea Herrera-Maldonado, ${ }^{5}$ Erika Paola García-Flores ${ }^{5}$

\begin{abstract}
Resumen
INTRODUCCIÓN: La detección temprana de la fibrosis quística mediante tamiz neonatal tiene una repercusión clínica positiva; su implementación es compleja y no existe un protocolo universal para llevarla a cabo. La cuantificación del tripsinógeno inmunorreactivo es el inicio de todos los algoritmos actuales de tamiz neonatal.

OBJETIVO: Reportar los resultados preliminares de la aplicación de un algoritmo de tamiz neonatal para fibrosis quística en la Secretaría de Salud de México.

MATERIAL Y MÉTODOS: Estudio retrospectivo de los resultados de la aplicación de un algoritmo de tamiz neonatal consistente en la cuantificación del tripsinógeno inmunorreactivo (IRT/IRT) seguida de la prueba del sudor para la detección de fibrosis quística en el marco del programa de tamiz metabólico neonatal de la Secretaría de Salud de México.

RESULTADOS: Se obtuvieron 1,267,122 muestras aptas para el procesamiento bioquímico de sangre en papel filtro de 1,273,727 recién nacidos; 3,216 muestras resultaron con valores sanguíneos de tripsinógeno inmunorreactivo superiores al percentil 99.5 . Solo en $54 \%$ de los casos se logró tomar la segunda muestra de tripsinógeno inmunorreactivo en el tiempo adecuado y se practicaron 1,787 pruebas de sudor. Se identificaron 202 casos altamente sugestivos de fibrosis quística (1:6,273 recién nacidos) que se enviaron para seguimiento médico. La gran cantidad de muestras extemporáneas favoreció el gran número de pruebas del sudor. Estos resultados permiten identificar las principales dificultades que enfrenta la implementación de este tamiz mediante el algoritmo IRT/IRT/ST.

CONCLUSIÓN: La incorporación de este primer algoritmo de detección de fibrosis quística al programa de tamiz neonatal en México representa un logro, susceptible de perfección.
\end{abstract}

PALABRAS CLAVE: Tamiz neonatal; fibrosis quística; CFTR; tripsinógeno inmunoreactivo; IRT.

Abstract

INTRODUCTION: Early detection of cystic fibrosis (CF) by newborn screening (NBS) has a positive effect on the clinical outcome of the disease however, its implementation is complex and there is no universal protocol. The quantification of immunoreactive trypsinogen (IRT) is used as initial biomarker of all current CF-NBS algorithms. OBJECTIVE: To present the preliminary results of the application of an algorithm for the NBS of CF in the Ministry of Health of Mexico.

MATERIALS AND METHODS: Retrospective study of the results of the application of an algorithm consisting of IRT/IRT quantification followed by sweat test (ST) for the detection of CF in the NBS program of the Ministry of Health of Mexico.

RESULTS: Filter paper blood samples were obtained from 1,273,727 newborns (NB), being 1,267,122 specimens suitable for biochemical processing; 3,216 samples showed IRT blood values above 95.5 percentile. Second IRT sample was obtained
${ }^{1}$ Unidad de Genética de la Nutrición, Instituto de Investigaciones Biomédicas, UNAM-Instituto Nacional de Pediatría SS, México.

2 Servicio de Neumología, Instituto Nacional de Pediatría, Secretaría de Salud, México.

${ }^{3}$ Laboratorio de Errores Innatos del Metabolismo y Tamiz, Instituto Nacional de Pediatría, Secretaría de Salud, México. ${ }^{4}$ TamizMas Screening Center de Químicos Maldonado, Mérida, Yucatán.

${ }^{5}$ Centro Nacional de Equidad de Género y Salud Reproductiva, Secretaría de Salud, México.

\section{Correspondencia}

Erika Paola García Flores

erika.garcia@salud.gob.mx

Este artículo debe citarse como Ibarra González I, Gutiérrez Morales G, Vela Amieva M, Castillo Mogel JA, Herrera Pérez LA, Caamal Parra G, Herrera Maldonado N, García Flores EP. Retos y oportunidades en la implementación del tamiz neonatal para fibrosis quística.Acta Pediatr Mex. 2018;SI(39):35S-46S. 
only in 54\% suspicious NB. 1,787 ST were performed and 202 highly CF suggestive cases $(1: 6,273 \mathrm{NB})$ were detected and sent to medical follow-up. The high number of extemporaneous samples contributes significantly to the high number of ST. Our results allow us to identify the main difficulties of the CF NBS implementation using the IRT/IRT/ST algorithm.

CONCLUSION: The incorporation of this first NBS algorithm for CF detection in Mexico represents an achievement however, it is necessary to improve it.

KEYWORDS: Newborn screening, cystic fibrosis, CFTR, immunoreactive trypsinogen, IRT.

\section{INTRODUCIÓN}

La fibrosis quística es una enfermedad multisistémica caracterizada por la infección crónica de las vías respiratorias superior e inferior que conduce a bronquiectasias y enfermedad pulmonar terminal. Tiene una herencia autosómica recesiva y es la enfermedad monogénica más frecuente en la población caucásica; se estima que afecta incluso a 1:1,000 recién nacidos europeos ${ }^{1}$ y 1:2,000 recién nacidos en Estados Unidos. $^{2}$ Es poco frecuente en poblaciones de la India (1:40,000 a 100,000 recién nacidos) y Japón (1:350,000 a 1:1,000,000 recién nacidos) (WHO). ${ }^{3}$ En población hispana de California, la prevalencia al nacimiento es de 1:9,259 recién nacidos. ${ }^{4,5}$ La fibrosis quística se debe a defectos en el gen CFTR (por sus siglas en inglés cystic fibrosis transmembrane conductance regulator) ubicado en el brazo largo del cromosoma 7 y cuyo producto génico es la proteína CFTR. El funcionamiento defectuoso de este gen altera el transporte de cloro y sodio en las células secretoras epiteliales, que se expresan en diversos órganos de la economía corporal que originan que las secreciones se tornen viscosas, se deshidraten y que contribuyan a las diversas manifestaciones según el órgano diana afectado. $^{4,6}$ (Figura 1)

El diagnóstico clínico de la fibrosis quística, en la mayoría de los individuos no detectados

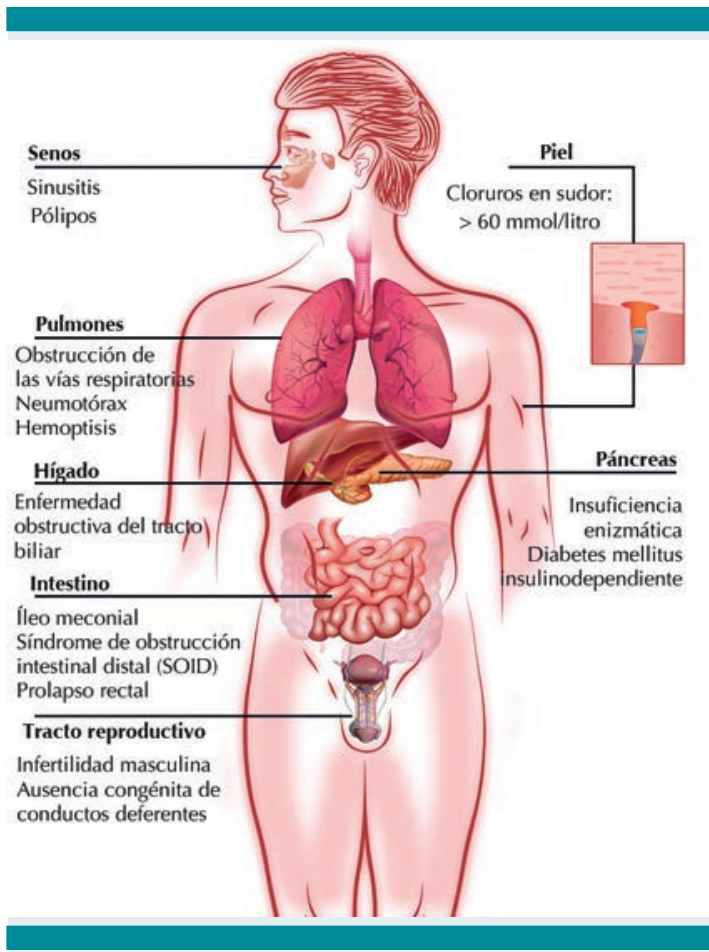

Figura 1. Esquema de los principales órganos y sistemas afectados en la fibrosis quística.

por el tamiz neonatal, se basa en una tríada caracterizada por infecciones sinopulmonares recurrentes, esteatorrea y retraso del crecimiento. En los pulmones, el defecto en el transporte de cloro conduce a mayor absorción de sodio, disminución de la secreción de agua y mayor reabsorción del líquido periciliar. ${ }^{6}$ El resultado es la disminución del volumen de líquido en las 
superficies de las vías respiratorias, formación de moco espeso y viscoso, ineficaz para el aclaramiento mucociliar; esto provoca una migración intensa de neutrófilos y origina la liberación de elastasa y grandes cantidades de ADN, como resultado de su degradación, lo que hace que el moco sea aún más viscoso y que conduzca a la aparición de sustancias proinflamatorias, que causan predisposición a la colonización e infección de las vías respiratorias y formación de bronquiectasias, creando un círculo vicioso que es causa importante del deterioro de la función pulmonar en estos pacientes. ${ }^{7}$

Debido a la obstrucción de los canalículos pancreáticos por las secreciones viscosas, la falta de funcionamiento de los canales de cloruro en las células epiteliales, las enzimas secretadas en los acinos no alcanzan el duodeno, lo que conduce a una digestión deficiente y malabsorción de macro y micronutrientes que causa desnutrición proteico-calórica. La insuficiencia pancreática coexiste desde el nacimiento en aproximadamente $75 \%$ de los individuos con fibrosis quística, en 80 a $85 \%$ al final del primer año de vida y en $90 \%$ en la edad adulta. ${ }^{8}$ Puesto que es una enfermedad multisistémica ${ }^{9}$ pueden encontrarse otras manifestaciones clínicas (Cuadro 1). En condiciones normales, en las glándulas sudoríparas el sodio (seguido de iones cloruro) se reabsorbe rápidamente desde la luz ductal, a través de los canales apicales de sodio y el CFTR. En pacientes con fibrosis quística la falta en el funcionamiento de CFTR restringe la reabsorción de cloruro, lo que limita la cantidad de sal que puede reutilizarse. Puesto que no existe otra ruta para la reabsorción eficaz del cloruro en el conducto, el sodio también se absorbe poco y el sudor que emerge en la superficie de la piel tiene un alto contenido de sodio. ${ }^{10}$

\section{Tamiz neonatal para fibrosis quística}

Existen reportes que demuestran que la detección temprana de la fibrosis quística mediante tamiz neonatal tiene un efecto positivo en los resulta-
Cuadro 1. Principales manifestaciones clínicas de la fibrosis quística, por órganos y aparatos afectados

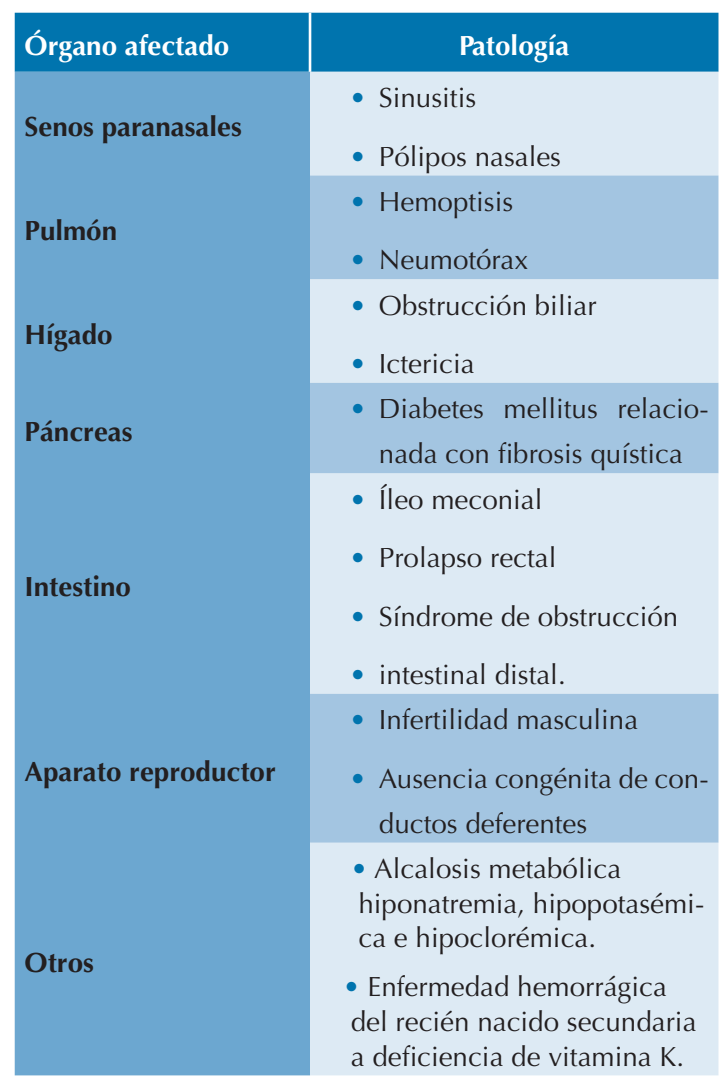

dos clínicos a corto y largo plazo., ${ }^{411-14}$ Existen diversas guías para la implementación de ese tamiz. ${ }^{15-18}$ Lo anterior ha conducido, desde hace más de tres décadas, a que diferentes programas de tamiz neonatal incorporen la detección de fibrosis quística, sobre todo en Europa y Estados Unidos. En la actualidad, todos los programas de tamiz neonatal para fibrosis quística comparten la misma prueba inicial, que consiste en la cuantificación de tripsinógeno inmunorreactivo (immunoreactive tripsinogen IRT, por sus siglas en inglés, en la nomenclatura internacional) en muestras de sangre impregnadas en papel filtro (tarjetas de Guthrie).

La implementación del tamiz neonatal para fibrosis quística es compleja en diversos sentidos: consume tiempo del personal de salud, 
genera falsos positivos, descubre portadores de la enfermedad, genera angustia en los padres y como todo programa de tamiz neonatal, puede tener casos falsos negativos. En la actualidad no existe un protocolo universal de tamiz neonatal para fibrosis quística y cada uno de ellos tiene ventajas y riesgos que hay que contemplar. 2,15,16 El objetivo de esta comunicación es mostrar los resultados preliminares de la aplicación de un algoritmo de tamiz neonatal para fibrosis quística en México.

\section{Fundamentos de la confirmación diagnóstica de la fibrosis quística}

Es indispensable confirmar el diagnóstico de fibrosis quística en el momento oportuno para evitar pruebas innecesarias, proveer el tratamiento adecuado, asesoramiento genético y asegurar el acceso a servicios especializados. El diagnóstico de fibrosis quística se basa en criterios clínicos (fenotipo) sugerentes o antecedente familiar y se corrobora al demostrar la disfunción del CFTR. EI patrón de referencia del diagnóstico de fibrosis quística sigue siendo el estudio de cloruros en sudor. La prueba del sudor (sweat test, ST por sus siglas en inglés), descrita por primera vez por Gibson y Cook en 1959, se basa en el estímulo mediante iontoforesis con pilocarpina, en un área de la piel asignada por el experto, seguida de la recolección y análisis de la concentración de cloruros en sudor. ${ }^{16,19}$

En la mayor parte de los países industrializados las técnicas de biología molecular para el análisis del gen CFTR se han incorporado a los algoritmos de tamiz neonatal de la fibrosis quística y generalmente se estudia el panel recomendado por el American College of Medical Genetics (ACMG) que incluye las 23 variantes más frecuentes en Estados Unidos; sin embargo, los paneles de variantes se deben ajustar y diseñar de acuerdo con las características genéticas de la población de estudio. ${ }^{20}$

\section{MATERIALES Y MÉTODOS}

Estudio retrospectivo y descriptivo de recién nacidos en la República Mexicana a quienes se les aplicó un algoritmo de tamiz para la detección de fibrosis quística en el marco del programa de tamiz metabólico neonatal de la Secretaría de Salud.

Del 1 de enero de 2017 al 31 de mayo de 2018 se llevó a cabo el tamiz neonatal en 1,273,727 niños nacidos en las unidades de la Secretaría de Salud de México.

Puesto que el tamiz neonatal es una acción obligatoria para todas las unidades en las que se da atención perinatal, de conformidad con el Artículo 61, Fracción II de la Ley General de Salud, con la Norma Técnica Mexicana NTM-321-1988 y con las Normas Oficiales Mexicanas NOM034-SSA2-2013 y NOM-007-SSA2-2016,,21-24 solo se consideró el consentimiento informado verbal para la aceptación del tamiz neonatal. Se tomaron cinco gotas de sangre del talón del recién nacido, que se recolectaron en papel de filtro estandarizado (tarjetas de Guthrie). Después de la recolección, los especímenes se enviaron, por mensajería, al laboratorio procesador contratado por el Centro Nacional de Equidad y Género y Salud Reproductiva (CNEGSR), que es la unidad de la Secretaría de Salud responsable de las Políticas Nacionales de los programas de Salud Materna y Perinatal entre los que se incluye el tamiz neonatal.

\section{Estrategia empleada}

Se utilizó la estrategia IRT/IRT/ST. Cuando se encontró un valor de tripsinógeno inmunorreactivo superior al percentil 99.5 e inferior a $97 \mathrm{ng} / \mathrm{mL}$ se repitió la determinación en el mismo espécimen y si la segunda determinación persistió elevada, se notificó y solicitó una segunda muestra de sangre, misma que debía tomarse, a más tardar, 
en la cuarta semana de vida del recién nacido. Si la segunda muestra de sangre tenía una concentración de tripsinógeno inmunorreactivo mayor de $60 \mathrm{ng} / \mathrm{mL}$, se solicitaba una prueba del sudor. En los casos en que la primera muestra el tripsinógeno inmunorreactivo fue mayor de $97 \mathrm{ng} /$ $\mathrm{mL}$ (valor crítico), el recién nacido sospechoso se envió directamente a la toma de prueba del sudor. Los casos con concentración elevada o muy elevada de tripsinógeno inmunorreactivo y prueba del sudor positiva se consideraron altamente sugestivos y se recomendó la referencia inmediata. En los recién nacidos con antecedente de íleo meconial se recomendó el envío inmediato al hospital correspondiente.

\section{Cuantificación del tripsinógeno inmunorreactivo en sangre neonatal}

El tripsinógeno inmunorreactivo se midió mediante ensayo inmunofluorométrico a tiempo resuelto con un instrumento General Screening Procesor GSP ${ }^{\circledR}$ y kits comerciales (Perkin Elmer, Waltham, MA).

\section{Realización de pruebas del sudor (ST)}

Las pruebas de sudor se realizaron por conductividad con los equipos Macroduct ${ }^{\circledR}$ (Webster Sweat Collection System 3700-SYS, Wescor) o Nanoduct ${ }^{\circledR}$ (Wescor Neonatal Sweat Analysis System Model 1030), siguiendo las instrucciones del fabricante y lo señalado en la bibliografía. ${ }^{25}$

Técnica utilizada con el Macroduct ${ }^{\circledR}$ (Webster Sweat Collection System 3700-SYS, Wescor): después de limpiar la piel con agua y secarla, se estimula la piel para favorecer la producción de sudor mediante iontoforesis con pilocarpina (almohadillas de Piloge ${ }^{\circledR}$ ) con una corriente máxima de $1.5 \mathrm{~mA}$, proveniente de una fuente eléctrica con batería y una duración de 5 minutos. Posteriormente se limpió y secó la piel, se colocó un disco recolector cóncavo sobre el área estimulada, con una apertura central que conecta con un capilar-plástico de $0.6 \mathrm{~mm}$ de diámetro en forma de espiral que colecta el sudor de la superficie de la piel en un tiempo no mayor de 30 minutos con una concentración mínima de $15 \mu \mathrm{L}$ para el análisis de la muestra. El cloruro y el sodio del sudor se midieron mediante un procedimiento de conductividad estándar. Los valores de referencia con este método son: positivo mayor de $60 \mathrm{mmol} / \mathrm{L}$; indeterminado 30-60 mmol/L; negativo menos de $30 \mathrm{mmol} / \mathrm{L}$.

Técnica con Nanoduct (Wescor Neonatal Sweat Analysis System Model 1030): después de limpiar la piel con alcohol al 70\% y agua destilada, se colocó un portaelectrodo-sensor en la extremidad del recién nacido, previa estimulación mediante iontoforesis con discos de geles de pilocarpina (Pilogel ${ }^{\circledR}$ ) con una corriente nominal de $0.5 \mathrm{~mA}$ por 2.5 minutos. Después de un tiempo cercano a 8 minutos se efectuó la lectura directa del resultado en la pantalla del equipo. La conductividad se midió durante la recolección de sudor. Los valores de referencia con este método son: positivo más de $80 \mathrm{mmol} / \mathrm{L}$; indeterminado $60-80 \mathrm{mmol} / \mathrm{L}$; negativo menos de $60 \mathrm{mmol} / \mathrm{L}$.

\section{Análisis estadístico}

Los datos de peso, sexo, edad gestacional (producto de término $\geq 37$ semanas de gestación o pretérmino menos de 37 semanas de gestación), estado de procedencia y estado de salud (sano o hospitalizado) se obtuvieron de la ficha de identificación de cada recién nacido y se consignaron en el SYSDQM ${ }^{\circledR}$. La edad de los recién nacidos se calculó al momento de la toma.

Se calculó la prevalencia al nacimiento y la tasa de falla (no localizados por todas las causas/ total de tamizados por 100,000). Se utilizó el programa de acceso libre Comprehensive $R$ Archive Network (https://cran.r-project.org). Para 
comparar las diferencias entre los grupos se utilizó la prueba de Mann-Whitney. Se consideró significación estadística un valor de $\mathrm{p}<0.05$.

\section{RESULTADOS}

De 1,273,727 recién nacidos tamizados, resultaron adecuadas para el procesamiento bioquímico 1,267,122 muestras, de las que 618,601 correspondieron a niñas $(48.82 \%)$ y 643,296 a niños (50.77\%). En 5,225 (0.42\%) muestras la ficha de identificación no contenía el dato de sexo o este fue señalado como ambiguo. Se registraron 68,382 recién nacidos prematuros (menos de 37 semanas de gestación), correspondientes a $5.40 \%$. El $2.2 \%$ de las muestras se obtuvieron antes del tercer día de vida extrauterina, $78.2 \%$ entre el tercero y quinto y $19.6 \%$ en mayores de 5 días de vida.

La distribución de los valores de las concentraciones en sangre en papel filtro de la población tamizada se muestran en la Figura 2. En la Figura 3 se encuentran las concentraciones de

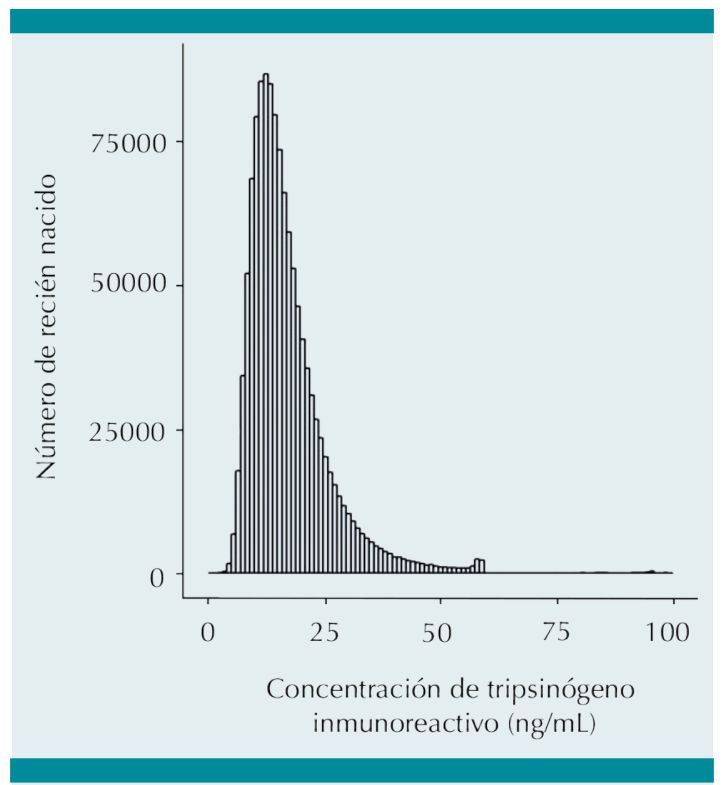

Figura 2. Distribución de las concentraciones de IRT en la población analizada n=1,000,000. tripsinógeno inmunorreactivo en papel filtro, dependiendo de las semanas de vida de los recién nacidos al momento de la toma del tamiz.

El panorama general de los resultados se expone en la Figura 4, en donde se observa que 3,210 muestras tuvieron concentraciones sanguíneas elevadas de tripsinógeno inmunorreactivo ( $>60$ $\mathrm{ng} / \mathrm{mL}$ ) y $1,263,906$ muestras tuvieron valores normales. En total, se realizaron 1,787 pruebas del sudor y como resultado, se identificaron 202 casos altamente sugestivos de fibrosis quística, mismos que se enviaron para seguimiento médico.

En el Cuadro 2 se reporta la cantidad casos sospechosos por estado, los casos altamente sugestivos y la estimación de la prevalencia al nacimiento de la enfermedad. En el Cuadro 3 se enlistan los principales problemas que se registraron en la implementación del tamiz para fibrosis quística.

\section{DISCUSIÓN}

El objetivo de los programas de tamiz neonatal para fibrosis quística se dirige a identificar y localizar al mayor número de recién nacidos afectados con la enfermedad para ofrecerles la oportunidad de ser tratados tempranamente con la finalidad de mejorar su estado de salud, con el mínimo de efectos adversos y con un costo asumible para el servicio de salud. ${ }^{26}$

Los algoritmos de tamiz neonatal para fibrosis quística en el mundo son muy variados y para su elaboración hay que tomar en cuenta múltiples factores económicos y sociales, así como las características genéticas de la población. Esta heterogeneidad no es necesariamente una debilidad porque los protocolos de tamiz neonatal deben adaptarse a las circunstancias locales. ${ }^{27}$ Si bien los beneficios de la detección neonatal y el tratamiento temprano de la fibrosis quística son conocidos, ${ }^{17,28,29}$ son pocos los países con economías emergentes que lo realizan. ${ }^{30}$ En esta 


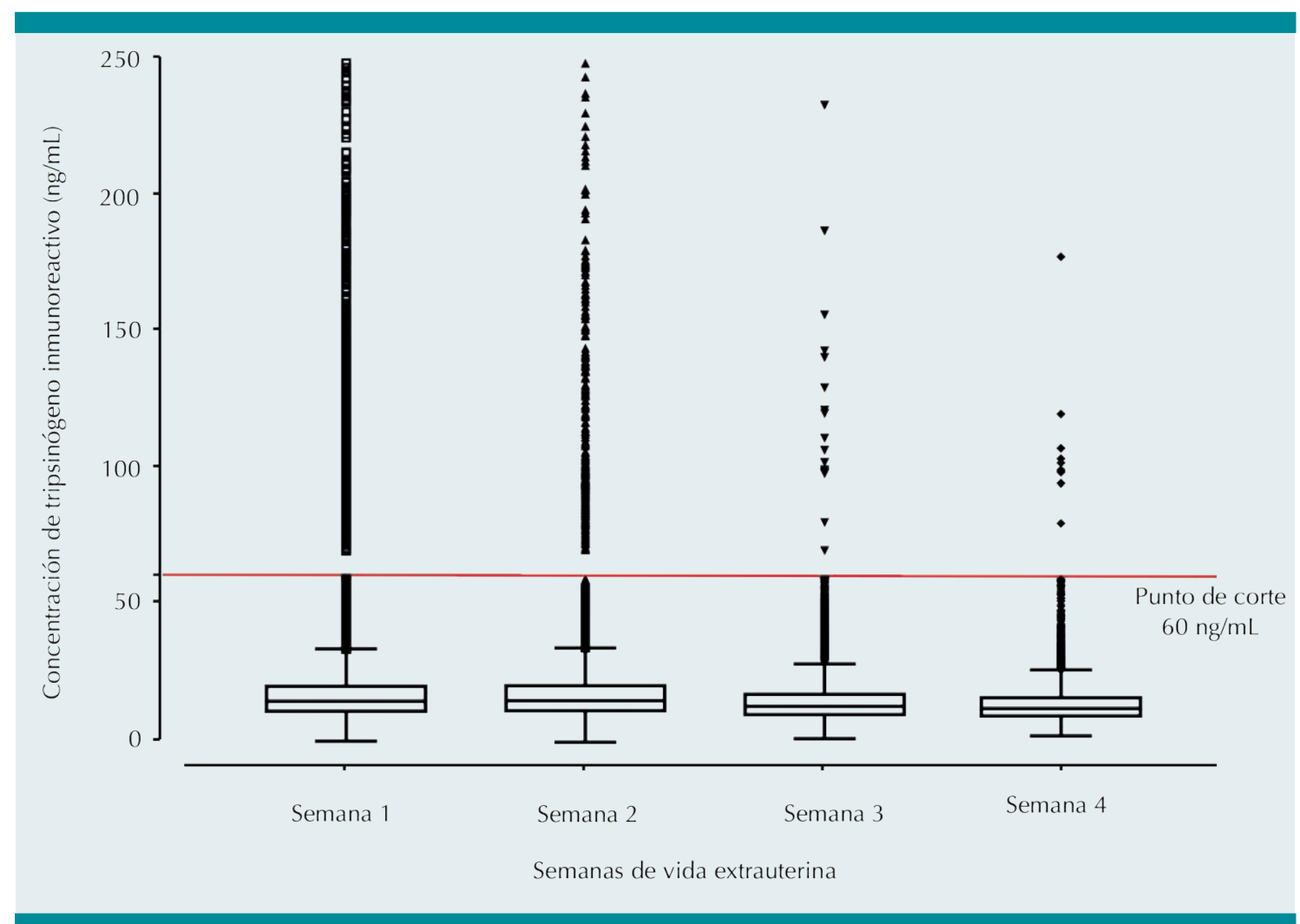

Figura 3. Concentraciones sanguíneas de IRT en las muestras de sangre por semana de edad al momento de la toma del tamiz $n=1,000,000$.

investigación se reporta, por primera vez, la experiencia de la implementación de un programa de tamiz neonatal a nivel nacional para fibrosis quística y los resultados son relevantes puesto que se obtuvieron en un país en vías de industrialización con un complejo sistema de salud y con un elevado número anual de nacimientos, lo que significa un reto para cualquier programa de tamiz neonatal. ${ }^{31,32}$

El uso del algoritmo IRT/IRT/ST permitió detec$\operatorname{tar} 202$ casos altamente sugestivos de fibrosis quística, con una tasa de prevalencia estimada al nacimiento de 16 por 100,000 recién nacidos tamizados (1:6,273 recién nacidos), con un máximo de 35 por 100,000 recién nacidos en el Estado de México (1:2,895 recién nacidos). Esta cifra no es la prevalencia real al nacimiento, sino que es la definición operacional del algoritmo y representa los casos con elevada probabilidad de tener la enfermedad que requieren ser referidos a evaluación médica especializada. Una limitante de este estudio es que no contó con la retroalimentación de las unidades médicas que llevan a cabo el seguimiento de los casos emanados del programa de tamiz neonatal, lo que es indispensable para poder contar con el número verdadero de pacientes y de casos falsos positivos. En el estado de Baja California Sur no se detectó ningún caso, sin embargo, la cantidad de tamizados es aún baja (6,272 recién nacidos tamizados). En el caso de Yucatán en el periodo analizado no se registraron casos; sin embargo, se sabe que la fibrosis quística en ese estado tiene 


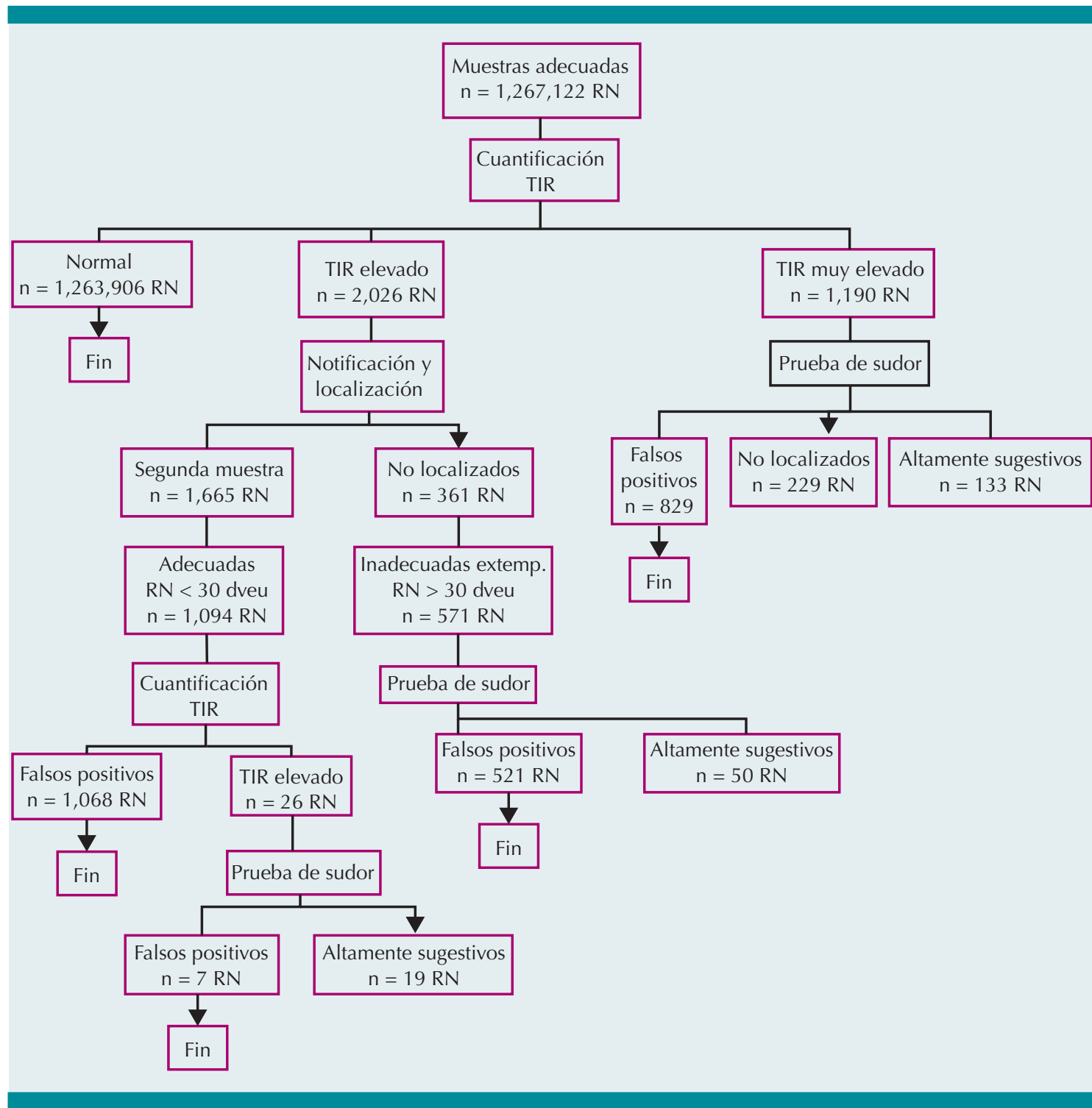

Figura 4. Panorama general de los resultados.

una prevalencia de 1:13,724. ${ }^{33}$ En Campeche, Coahuila y Zacatecas tampoco se han registrado casos, pero en estos estados la tasa de falla es elevada. Cuadro 2

La distribución de las concentraciones sanguíneas de tripsinógeno inmunorreactivo en los recién nacidos mexicanos (Figura 2) es semejante a la reportada en la bibliografía., 2,34 También se observa que tal como se describió, los valores de tripsinógeno inmunorreactivo son edad-dependientes (Figura 3), por eso con el transcurso del tiempo las concentraciones de tripsinógeno inmunorreactivo disminuyen. ${ }^{2,35}$ Este último punto es muy relevante puesto que señala claramente una de las debilidades del trip- 
Ibarra-González I, et al. Tamiz neonatal para fibrosis quística

Cuadro 2. Recién nacidos tamizados, número de muestras adecuadas, casos sospechosos, confirmados y prevalencia al nacimiento de fibrosis quística en México

\begin{tabular}{|c|c|c|c|c|c|c|c|c|}
\hline Entidad & $\begin{array}{c}\mathbf{R N} \\
\text { tamizados }\end{array}$ & $\begin{array}{c}\text { Muestras } \\
\text { adecuadas }\end{array}$ & $\begin{array}{c}\text { Muestras } \\
\text { sospechosas }\end{array}$ & Casos & $\begin{array}{c}\text { Falsos } \\
\text { positivos }\end{array}$ & \begin{tabular}{|c|} 
No \\
localizados
\end{tabular} & $\begin{array}{c}\text { Prevalencia } \\
\text { estimada al } \\
\text { nacimiento } \\
(1: \text { RN })\end{array}$ & $\begin{array}{c}\text { Tasa de } \\
\text { falla* por } \\
100,000 \\
\text { RN }\end{array}$ \\
\hline Aguascalientes & 16,896 & 16,878 & 33 & 5 & 27 & 1 & 3,376 & 5.9 \\
\hline $\begin{array}{l}\text { Baja California } \\
\text { Norte }\end{array}$ & 24,341 & 24,290 & 29 & 4 & 22 & 3 & 6,073 & 12.4 \\
\hline $\begin{array}{l}\text { Baja California } \\
\text { Sur }\end{array}$ & 6,272 & 6,245 & 9 & 0 & 9 & 0 & 0 & 0 \\
\hline Campeche & 11,683 & 11,646 & 12 & 0 & 8 & 4 & 0 & 34.3 \\
\hline Chiapas & 59,709 & 59,204 & 68 & 6 & 47 & 15 & 9,867 & 25.3 \\
\hline Chuhuahua & 25,699 & 25,593 & 34 & 2 & 27 & 5 & 12,797 & 19.5 \\
\hline $\begin{array}{l}\text { Ciudad de } \\
\text { México }\end{array}$ & 42,795 & 42,694 & 210 & 13 & 143 & 54 & 3,284 & 126.5 \\
\hline Coahuila & 22,634 & 22,465 & 33 & 0 & 30 & 3 & 0 & 13.4 \\
\hline Colima & 7,657 & 7,647 & 17 & 1 & 12 & 4 & 7,647 & 52.3 \\
\hline Durango & 20,705 & 20,583 & 39 & 2 & 31 & 6 & 10,292 & 29.2 \\
\hline $\begin{array}{l}\text { Estado de Méx- } \\
\text { ico }\end{array}$ & 150,948 & 150,558 & 878 & 52 & 611 & 215 & 2,895 & 142.8 \\
\hline Guanajuato & 101,602 & 100,935 & 214 & 10 & 178 & 26 & 10,094 & 25.8 \\
\hline Guerrero & 61,337 & 61,212 & 139 & 11 & 113 & 15 & 5,565 & 24.5 \\
\hline Hidalgo & 39,430 & 39,385 & 105 & 3 & 94 & 8 & 13,128 & 20.3 \\
\hline Jalisco & 77,353 & 77,051 & 136 & 16 & 105 & 15 & 4,816 & 19.5 \\
\hline Michoacán & 64,516 & 64,114 & 149 & 8 & 118 & 23 & 8,014 & 35.9 \\
\hline Morelos & 22,739 & 22,727 & 45 & 4 & 34 & 7 & 5,682 & 30.8 \\
\hline Nayarit & 9,981 & 9,940 & 18 & 3 & 15 & 0 & 3,313 & 0 \\
\hline Nuevo León & 31,997 & 31,928 & 27 & 7 & 16 & 4 & 4,561 & 12.5 \\
\hline Oaxaca & 48,151 & 47,848 & 153 & 10 & 108 & 35 & 4,785 & 73.1 \\
\hline Puebla & 96,041 & 95,773 & 344 & 14 & 263 & 67 & 6,841 & 70.0 \\
\hline Querétaro & 24,351 & 23,951 & 60 & 8 & 45 & 7 & 2,994 & 29.2 \\
\hline Quintana Roo & 19,930 & 19,772 & 32 & 2 & 20 & 10 & 9,886 & 50.6 \\
\hline San Luis Potosí & 28,759 & 28,644 & 32 & 1 & 28 & 3 & 28,644 & 10.5 \\
\hline Sinaloa & 26,928 & 26,908 & 15 & 5 & 9 & 1 & 5,382 & 3.7 \\
\hline Sonora & 21,318 & 21,273 & 13 & 1 & 11 & 1 & 21,273 & 4.7 \\
\hline Tabasco & 31,556 & 31,235 & 27 & 1 & 21 & 5 & 31,235 & 16.0 \\
\hline Tamaulipas & 35,588 & 35,484 & 35 & 4 & 30 & 1 & 8,871 & 2.8 \\
\hline Tlaxcala & 19,524 & 19,506 & 103 & 6 & 67 & 30 & 3,251 & 153.8 \\
\hline Veracruz & 80,306 & 78,739 & 100 & 3 & 85 & 12 & 26,246 & 15.2 \\
\hline Yucatán & 22,715 & 22,715 & 50 & 0 & 48 & 2 & 0 & 8.8 \\
\hline Zacatecas & 20,266 & 20,179 & 57 & 0 & 50 & 7 & 0 & 34.7 \\
\hline Total & $1,273,727$ & $1,267,122$ & 3,216 & 202 & 2,425 & 589 & 6,273 & 46.5 \\
\hline
\end{tabular}

* La tasa de falla comprende al número de no localizados, independiente de la causa e incluye a los fallecidos (no localizados/ total de tamizados x 100,000 recién nacidos tamizados). 
Cuadro 3. Principales obstáculos y dificultades detectadas en la implementación del tamiz neonatal para fibrosis quística mediante el algoritmo IRT/IRT/ST

\begin{tabular}{|c|c|}
\hline Fase del tamiz & Obstáculos y dificultades \\
\hline Preanalíticas & $\begin{array}{l}\text { 1. Tardanza en el tránsito de la } \\
\text { muestra, desde su toma hasta la } \\
\text { Ilegada al laboratorio. } \\
\text { 2. Elevado número de muestras } \\
\text { iniciales tomadas en forma tar- } \\
\text { día después de } 15 \text { días de vida } \\
\text { extrauterina. } \\
\text { 3. Falta de capacitación del } \\
\text { personal de salud que toma la } \\
\text { muestra, para enviar los casos } \\
\text { de RN con íleo meconial a } \\
\text { una evaluación directa por el } \\
\text { neumólogo pediatra. }\end{array}$ \\
\hline Analíticas & $\begin{array}{l}\text { 1. Baja especificidad de IRT, } \\
\text { que genera un elevado número } \\
\text { de falsos positivos. }\end{array}$ \\
\hline Postanalíticos & $\begin{array}{l}\text { 1. Alta tasa de falla de localiza- } \\
\text { ción de los casos sospechosos. } \\
\text { 2. El algoritmo y las muestras. } \\
\text { inadecuadas extemporáneas } \\
\text { generan un elevado número } \\
\text { de ST. } \\
\text { 3. Ausencia de un sistema esta- } \\
\text { blecido de seguimiento a corto, } \\
\text { mediano y largo plazo de los } \\
\text { casos sospechosos. } \\
\text { 4. Ausencia de notificación ofi- } \\
\text { cial de casos falsos negativos. }\end{array}$ \\
\hline
\end{tabular}

sinógeno inmunorreactivo como biomarcador para la detección de la fibrosis quística y realza la necesidad de que las muestras de sangre de los recién nacidos se tomen tempranamente de preferencia antes del quinto día de vida para que, en caso de elevación, la segunda muestra pueda tomarse a tiempo, antes de la cuarta semana de vida, para ser procesada. El elevado número de muestras extemporáneas contribuye al alto número de pruebas del sudor que fue necesario tomar (Figura 4). La prueba del sudor es compleja; más allá de la destreza técnica para su realización, requiere una correcta interpretación y seguimiento clínico, de preferencia realizado en centros especializados que cuenten con neumólogos pediatras. ${ }^{16}$
Los resultados de esta investigación permiten identificar algunos obstáculos y dificultades en la fase preanalítica, analítica y postanalítica que enfrenta la implementación de este tamiz mediante el algoritmo IRT/IRT/ST (Cuadro 3). Este es el primer algoritmo que se usa en México y las experiencias nacionales e internacionales pueden contribuir a perfeccionarlo..$^{2,36,37}$ En otras regiones geográficas, parte de estos problemas se han resuelto mediante el fortalecimiento de la capacitación preanalítica, ${ }^{27}$ con el uso de biomarcadores adicionales, sobre todo la biología molecular del gen CFTR y, más recientemente, la incorporación de la proteína asociada a pancreatitis (PAP), ${ }^{38-40}$ y con el establecimiento de sistemas postanalíticos formales de seguimiento del tamiz neonatal. ${ }^{26,28,37,41}$

\section{CONCLUSIÓN}

La incorporación de la detección de fibrosis quística al programa de tamiz metabólico neonatal en México representa un logro, sin embargo, existen grandes áreas de oportunidad en cada una de las fases del tamiz. Considerando lo heterogéneo de las regiones del país, la complejidad del Sistema Nacional de Salud y la disponibilidad de recursos financieros y humanos en los servicios de salud, se identifica la necesidad de contar con alternativas que permitan corroborar el adecuado seguimiento y tratamiento.

Es necesario perfeccionar el algoritmo de detección actual mediante la incorporación de herramientas adicionales, como la biología molecular, para que se consiga la correcta definición de los casos y es indispensable la participación de personal capacitado en el seguimiento y control de las personas con fibrosis quística.

\section{REFERENCIAS}

1. Bobadilla JL, Macek M Jr, Fine JP, Farrell PM. Cystic fibrosis: a worldwide analysis of CFTR mutations--correlation with incidence data and application to screening. Hum Mutat. 2002;19(6):575-606. 
2. Therrell BL, Hannon WH, Hoffman G, Ojodu J, Farrell PM. Immunoreactive trypsinogen (IRT) as a biomarker for cystic fibrosis: challenges in newborn dried blood spot screening. Mol Genet Metab 2012;106(1):1-6. doi: 10.1016/j. ymgme.2012.02.013.

3. World Health Organization. The molecular genetic epidemiology of cystic fibrosis, http://www.who.int/genomics/ publications/en (2004, accessed 12 August 2017).

4. Coffey MJ, Whitaker V, Gentin N, Junek R, Shalhoub C, Nightingale S, Hilton J, Wiley V, Wilcken B, Gaskin KJ, Ooi CY. Differences in Outcomes between Early and Late Diagnosis of Cystic Fibrosis in the Newborn Screening Era. J Pediatr. 2017;181:137-145.e1. doi: 10.1016/j.jpeds.2016.10.045.

5. Kharrazi $M$, Yang J, Bishop T, Lessing $S$, Young $S$, Graham S, Pearl M, Chow H, Ho T, Currier R, Gaffney L, Feuchtbaum L; California Cystic Fibrosis Newborn Screening Consortium. Newborn screening for cystic fibrosis in $\mathrm{Ca}$ lifornia. Pediatrics 2015;136(6):1062-1072. doi: 10.1542/ peds.2015-0811.

6. Vankeerberghen, A., Cuppens, H., \& Cassiman, J.J. The cystic fibrosis transmembrane conductance regulator: An intriguing protein with pleiotropic functions. J Cyst Fibros, 2002;1(1):13-29. doi.org/10.1016/\$1569-1993(01)00003-0

7. De Rose V. Mechanisms and markers of airway inflammation in cystic fibrosis. Eur Respir J.2002;19(2):333-40. doi.org/10.1183/09031936.02.00229202

8. Evans AK, Fitzgerald DA, McKay KO. The impact of meconium ileus on the clinical course of children with cystic fibrosis. Eur Respir J. 2001;18(5):784-9. doi.org/10.1016/ S1569-1993(07)60174-X.

9. Paranjape, S. M., \& Mogayzel, P. J. Cystic Fibrosis. Pediatr Rev 2014, 35(5):194-205. doi.org/10.1542/pir.35-5-194

10. Rowe SM, Miller S, Sorscher EJ. Cystic fibrosis. N Engl J Med. 2005 12;352(19):1992-2001. doi.org/10.1007/9781-61779-120-8

11. Castellani C, Massie J, Sontag M, Southern KW. Newborn screening for cystic fibrosis. Lancet Respir Med. 2016;4(8):653-661. doi: 10.1016/S2213-2600(16)00053-9.

12. Rosenfeld M, Sontag MK, Ren CL. Cystic Fibrosis Diagnosis and Newborn Screening. Pediatr Clin North Am. 2016;63(4):599-615. doi: 10.1016/j.pcl.2016.04.004

13. Balfour-Lynn IM Newborn screening for cystic fibrosis: evidence for benefit. Arch Dis Child. 2008;93(1):7-10.

14. Tridello G, Castellani C, Meneghelli I, Tamanini A, Assael BM. Early diagnosis from newborn screening maximises survival in severe cystic fibrosis. ERJ Open Res. 2018 20;4(2). pii: 00109-2017. doi: 10.1183/23120541.001092017

15. Comeau AM1, Accurso FJ, White TB, Campbell PW 3rd, Hoffman G, Parad RB, Wilfond BS, Rosenfeld M, Sontag MK, Massie J, Farrell PM, O'Sullivan BP; Cystic Fibrosis Foundation. Guidelines for implementation of cystic fibrosis newborn screening programs: Cystic Fibrosis Foundation workshop report. Pediatrics. 2007;119(2):e495-518.
16. Farrell PM, White TB, Ren CL, Hempstead SE, Accurso F, Derichs N, Howenstine M, McColley SA, Rock M, Rosenfeld $M$, Sermet-Gaudelus I, Southern KW, Marshall BC, Sosnay PR. Diagnosis of Cystic Fibrosis: Consensus Guidelines from the Cystic Fibrosis Foundation. J Pediatr. 2017;181S:S4-S15. e1. doi: 10.1016/j.jpeds.2016.09.064.

17. Castellani C, Duff AJA, Bell SC, Heijerman HGM, Munck A, Ratjen F, Sermet-Gaudelus I, Southern KW, Barben J, Flume PA, Hodková P, Kashirskaya N, Kirszenbaum MN, Madge S, Oxley H, Plant B, Schwarzenberg SJ, Smyth AR, Taccetti G, Wagner TOF, Wolfe SP, Drevinek P. ECFS best practice guidelines: the 2018 revision. J Cyst Fibros. 2018;17(2):153178. doi: 10.1016/j.jcf.2018.02.006.

18. NHS Screening Programmes A Laboratory Guide to Newborn Screening in the UK for Cystic Fibrosis. Fourth Edition February 2014. www.newbornbloodspot.screening.nhs.uk

19. Vernooij-van Langen A, Dompeling E, Yntema JB, Arets B, Tiddens $\mathrm{H}$, Loeber $\mathrm{G}$, Dankert-Roelse J. Clinical evaluation of the Nanoduct sweat test system in the diagnosis of cystic fibrosis after newborn screening. Eur J Pediatr. 2015;174(8):1025-34. doi: 10.1007/s00431-015-2501-0.

20. Wiencek JR, Lo SF. Advances in the Diagnosis and Management of Cystic Fibrosis in the Genomic Era. Clin Chem. 2018 Jun;64(6):898-908. doi: 10.1373/clinchem.2017.274670.

21. Ley General de Salud (2007). Disponible: http://www. salud.gob.mx/unidades/cdi/legis/lgs/index-indice.htm.

22. Norma Técnica número 321 para la prevención del retraso mental producido por hipotiroidismo congénito. Diario Oficial de la Federación, 22/09/1988, México.

23. Norma Oficial Mexicana NOM-034-SSA2-2013, Para la prevención y control de los defectos al nacimiento. Diario Oficial de la Federación, 24/06/2014, México.

24. Norma Oficial Mexicana NOM-007-SSA2-2016, Para la atención de la mujer durante el embarazo, parto y puerperio, y de la persona recién nacida. Diario Oficial de la Federación, 01/04/2016, México.

25. Vernooij-van Langen A, Dompeling E, Yntema JB, Arets B, Tiddens $\mathrm{H}$, Loeber $\mathrm{G}$, et al. Clinical evaluation of the $\mathrm{Na}$ noduct sweat test system in the diagnosis of cystic fibrosis after newborn screening. Eur J Pediatr. 2015;174(8):102534. doi: 10.1007/s00431-015-2501-0.

26. Paz Valiñas L. Cribado neonatal de la fibrosis quística. Eficacia/efectividad y protocolos de implementación. Red Española de Agencias de Evaluación de Tecnologías y Prestaciones del SNS. Axencia de Avaliación de Tecnoloxías Sanitarias de Galicia; 2013. Informes de evaluación de tecnologías sanitarias.

27. Sarles J, Giorgi R, Berthézène $P$, Munck A, Cheillan D, Dagorn JC, et al. Neonatal screening for cystic fibrosis: comparing the performances of IRT/DNA and IRT/PAP. J Cyst Fibros. 2014;13(4):384-90. doi: 10.1016/j.jcf.2014.01.004.

28. Comeau AM, Parad R, Gerstle R, O'Sullivan BP, Dorkin HL, Dovey $M$ et al. Challenges in implementing a successful newborn cystic fibrosis screening program. J Pediatr. 2005;147(3 Suppl):S89-93. 
29. Grosse SD. Showing Value in Newborn Screening: Challenges in Quantifying the Effectiveness and Cost-Effectiveness of Early Detection of Phenylketonuria and Cystic Fibrosis. Healthcare (Basel). 2015;3(4):1133-57.

30. Therrell BL, Padilla CD, Loeber JG, Kneisser I, Saadallah A, Borrajo GJ, Adams J. Current status of newborn screening worldwide: 2015. Semin Perinatol. 2015;39(3):171-87. doi: 10.1053/j.semperi.2015.03.002.

31. Therrell BL Jr, Padilla CD. Newborn screening in the developing countries. Curr Opin Pediatr. 2018. doi: 10.1097/ MOP.0000000000000683.

32. Howson CP, Cedergren B, Giugliani R, Huhtinen P, Padilla CD, Palubiak CS, et al. Universal newborn screening: A roadmap for action. Mol Genet Metab. 2018;124(3):177-183. doi: 10.1016/j.ymgme.2018.04.009.

33. Ibarra-González I, Campos-Garcia FJ, Herrera-Pérez LDA, Martínez-Cruz P, Moreno-Graciano CM, Contreras-Capetillo SN, León-Burgos V, Maldonado-Solis FA, Alcántara-Ortigoza MA, González Del Angel A, Vela-Amieva $M$. Newborn cystic fibrosis screening in southeastern Mexico: Birth prevalence and novel CFTR gene variants. J Med Screen. 2017;1:969141317722808. doi: 10.1177/0969141317722808.

34. Cheillan D1, Vercherat M, Chevalier-Porst F, Charcosset M, Rolland MO, Dorche C. False-positive results in neonatal screening for cystic fibrosis based on a three-stage protocol (IRT/DNA/IRT): Should we adjust IRT cut-off to ethnic origin?. J Inherit Metab Dis. 2005;28(6):813-8.

35. Rodrigues R1, Magalhaes PK, Fernandes MI, Gabetta CS, Ribeiro AF, Pedro KP, et al. Neonatal screening for cystic fibrosis in São Paulo State, Brazil: a pilot study. Braz J Med Biol Res. 2009;42(10):973-8.
36. Lezana Fernández JL, Bustamante Sáenz A, Ovando Fonseca $\mathrm{JE}$, Boites Velarde R, Ruiz Gutiérrez HH (Editores). Fibrosis Quística. Guías clínicas para el diagnóstico y tratamiento. Intersistemas Editores, México D.F., 2015, 2a edición. 156 pp.

37. Hale JE, Parad RB, Dorkin HL, Gerstle R, Lapey A, O'Sullivan $B P$, et al. Cystic fibrosis newborn screening: using experience to optimize the screening algorithm. J Inherit Metab Dis. 2010;33(Suppl 2):S255-61. doi: 10.1007/s10545-010-9117-3

38. Haute Autorité de Santé. Place de la stratégie couplant les dosages de la trypsine immunoréactive (TIR) et de la protéine associée à la pancréatite (PAP) dans le dépistage systématique de la mucoviscidose en France. Haute Autorité de Santé - Service évaluation économique et santé publique; 2015.https://www.has-sante.fr/portail/upload/ docs/application/pdf/2015-06/place_de_la_strategie_couplant_les_dosages_de_la_tir_et_de_la_pap_dans_le_depistage_systematique_de_la_mucoviscidose_en_france.pdf.

39. Seror V, Cao C, Roussey M, Giorgi R. PAP assays in newborn screening for cystic fibrosis: a population-based cost-effectiveness study. J Med Screen 2016;23(2):62-9. https://doi. org/10.1177/0969141315599421.

40. Dankert-Roelse JE, Bouva MJ, Jakobs BS, Janssens HM, de Winter-de Groot KM, Schönbeck Y, et al. Newborn blood spot screening for cystic fibrosis with a four-step screening strategy in the Netherlands.J Cyst Fibros. 2018 Aug 23. pii: S1569-1993(18)30705-7. doi:10.1016/j.jcf.2018.07.008.

41. Sermet-Gaudelus I, Brouard J, Audrézet MP, Couderc Kohen L, Weiss L, Wizla N, et al. Guidelines for the clinical management and follow-up of infants with inconclusive cystic fibrosis diagnosis through newborn screening. Arch Pediatr. 2017;24(12):e1-e14. doi: 10.1016/j.arcped.2017.07.015. 